\title{
ROTATING PROGENITORS OF THE WOLF-RAYET BINARIES HD186943 AND HD90657
}

\author{
Jelena Petrovic ${ }^{1}$ and Norbert Langer ${ }^{1}$
}

We present rotating progenitor models for the WR+O binary systems HD186943 and HD90657 (van der Hucht, 2001) calculated with the evolutionary code described by Wellstein (2001). Due to rotation, the effective accretion onto the secondary star is reduced as it is spun-up close to its break-up velocity and thereby undergoes strong mass loss. We investigate the progenitor evolution of the two observed WR+O binary systems through stable mass transfer. We conclude that these systems evolved through Case A mass transfer as they have short orbital periods now (8...i. days), and the progenitor systems must have started with a shorter or equal period. We show that there has to be a significant mass loss from the binary system to produce $\mathrm{WR}+\mathrm{O}$ systems similar to the observed ones. The accretion efficiency of the secondary star in our rotating models is $\sim 10 \%$. We compare properties of the observed and modelled systems in Table 1.

We try to find out which fraction of the mass leaving the primary star is accumulated by the secondary star during a mass transfer event. Wellstein (2001) showed in his Case B models that rotation influences the accretion efficiency. When the secondary star is accreting matter from the primary it will spin up and start losing additional amounts of mass. Thus, the effective accretion rate is reduced due to rotation and the value of $\beta\left(M_{2, a c c} / M_{1, \text { lost }}\right)$ decreases.

We show in our models that this happens also in Case A systems. We calculated models of rotating systems with primaries of $M_{1}>50 \mathrm{M}_{\odot}$, initial mass ratios between $1.7 \ldots 2.0$ and with an initial orbital period of 6 days. These systems evolve through early Case A and Case AB mass transfer and form the $\mathrm{WR}+\mathrm{O}$ binary systems given in Table 1 . Systems are calculated for $1 / 6$ and $1 / 3$ of the WR mass loss rate proposed by Hamann et al. (1995), denoted further as $\mathrm{H} / 6$ and $\mathrm{H} / 3$.

During most of the fast phase of Case A mass

\footnotetext{
${ }^{1}$ Sterrenkundig Instituut, Princetonplein 5, $3584 \mathrm{CC}$ Utrecht, The Netherlands (petrovic@astro.uu.nl).
}

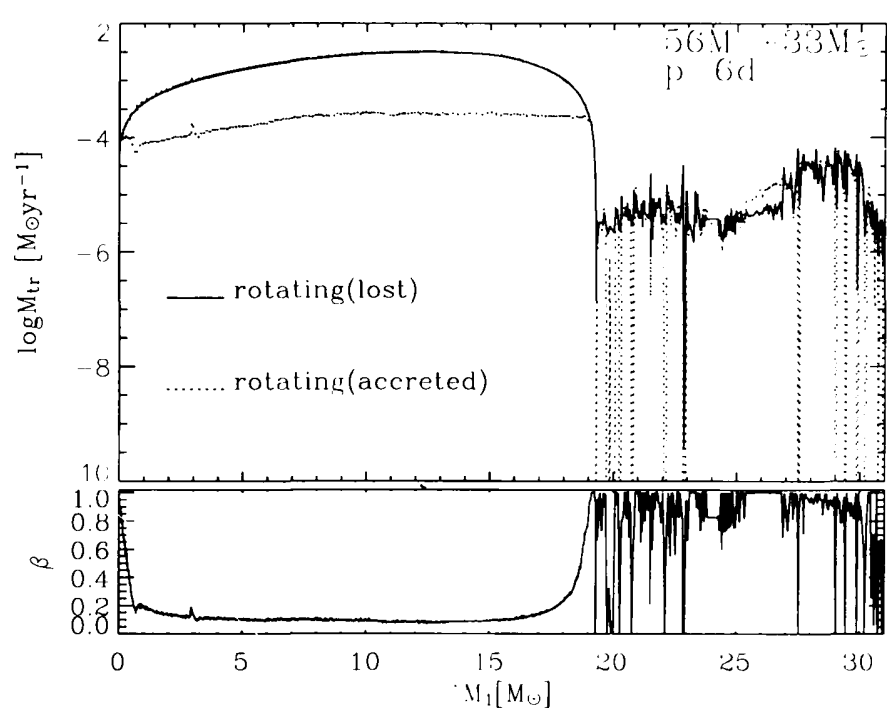

Fig. 1. Upper plot: mass transfer and accretion rate. Lower plot: change in accretion efficiency $\beta$.

transfer the effective mass accretion rate of the secondary is about one order of magnitude lower than the mass transfer rate. The mass transfer rate is about $\sim 10^{-3} \mathrm{M}_{\odot} \mathrm{yr}^{-1}$, the secondary spins up significantly and loses large amount of mass. When the mass transfer rate is $\sim 10^{-5}-10^{-6} \mathrm{MI}, \mathrm{yr}^{-1}$, the secondary is able to accrete almost everything from the primary, without spinning-up to close to the critical rotational velocity (slow phase (ase A).

Table 1. Observations \& models

\begin{tabular}{lccc}
\hline & $M_{\mathrm{wr}}^{5}\left(M_{\mathrm{wr}}^{1}\right)+M_{O}\left[M_{.2}\right]$ & $q$ & $p[\mathrm{~d}]$ \\
\hline$H D 186943$ & $17+36$ & 0.47 & 9.56 \\
$H D 90657$ & $19+37$ & 0.51 & 8.26 \\
$56+33^{H / 6}$ & $14.9(13.6)+42.1$ & 0.35 & 11.57 \\
$56+33^{H / 3}$ & $14.8(12.8)+39.0$ & 0.38 & 8.49 \\
$60+35^{H / 6}$ & $15.7(14.6)+45.1$ & 0.35 & 12.74 \\
$60+35^{H / 3}$ & $14.9(12.2)+42.4$ & 0.35 & $\mathbf{7 . 6 5}$ \\
\hline$M_{\mathrm{WR}}^{5} . M_{\mathrm{WR}}^{1}$ - mass when hydrogen surface abun-
\end{tabular}
dance in model is $\sim 5 \%$ and $\sim 1 \%$.

\section{REFERENCES}

Hamann W.-R., Koenterke L., Wossolowski U., 1905. A\&A 299, 151

van der Hucht, 2001, NewAR, v.45, p.135-23:2

Wellstein, S., Langer. ․, 2001, Ad A. v.369, p.939-9.5!

Wellstein S., 2001, PhD thesis. Potsdam Iniversity. 